\title{
TreFoil Factor 1 (TFF1/pS2) Deficiency Activates the Unfolded Protein Response
}

\author{
Luis-Fernando Torres, ${ }^{2}$ Sherif M. Karam, ${ }^{3}$ Corinne Wendling, ${ }^{1}$ Marie-Pierre Chenard, ${ }^{1}$ \\ David Kershenobich, ${ }^{2}$ Catherine Tomasetto, ${ }^{1}$ and Marie-Christine Rio ${ }^{1}$ \\ ${ }^{1}$ Institut de Génétique et de Biologie Moléculaire et Cellulaire (IGBMC), Illkirch Cedex, C.U. de Strasbourg, \\ France \\ ${ }^{2}$ Gastroenterology Department, Instituto Nacional de Ciencias Médicas y de la Nutricion "Salvador Zubiran" \\ (INCMNSZ), Distrito Federal, México \\ ${ }^{3}$ Department of Anatomy, Faculty of Medicine \& Health Sciences, United Arab Emirates University, Al Ain, \\ United Arab Emirates \\ Accepted April 20, 2002
}

\begin{abstract}
Background: The trefoil factor 1 (TFFl/pS2) is a secreted gastrointestinal peptide that is often altered or lost in human gastric cancers. Consistently, mouse TFFl deficiency leads to antropyloric tumors.

Materials and Methods: To investigate the gene expression alterations in response to the lack of TFFl, we performed differential expression analyses of TFF1 null antropyloric tumors using an array containing 588 cDNAs. Results: Using total and enriched probes, 22 genes were found to be up-regulated. The identification of the genes for endoplasmic reticulum (ER)-resident GRP78, ERp72, and p58IPK proteins connected TFFl deficiency to the unfolded protein response (UPR). Accordingly, CHOP10, a transcription factor induced early in response to ER stress, and the pleiotropic Clusterin, involved in protein
\end{abstract}

folding, were also overexpressed. Northern blot analyses of 8 weeks and 1 year TFFl null tumors confirmed that GRP78, ERp72, p58IPK, CHOP10, and Clusterin overexpression is a common and permanent feature shared by all TFF1 null antropyloric tumors. Finally, consistent with UPR, ultrastructural analyses showed that tumor rough ER was enlarged and contained dense material, supporting the hypothesis that TFFl deficiency leads to the accumulation of misfolded proteins in the ER.

Conclusion: Together, our data provide the first evidence of a relationship between a member of the TFF family and the ER machinery. Whereas to date TFFl is believed to act as an extracellular molecule, our results suggest a possible additional function for TFFl in protein folding and/or secretion.

\section{Introduction}

In mammals, the trefoil factor family (TFF) is composed of three small secreted peptides, namely TFF1 (formerly pS2), TFF2 (formerly SP for spasmolytic polypeptide), and TFF3 (formerly ITF for intestinal Trefoil factor) characterized by six conserved cysteine residues involved in disulfide bonds forming the TFF domain. One copy of the TFF domain is present in TFF1 and TFF3, whereas two in tandem domains are present in TFF2. An acidic carboxy terminal region is also conserved in all members of this family. Within this domain a free cysteine, present in TFF1 and TFF3, can promote homo-and heterodimerization (for review see: 1-3).

TFFl is highly expressed in the normal stomach and the protein is secreted in gastric juice (4). In the gastrointestinal (GI) tract, TFFs are expressed and secreted in discrete complementary regions. Except

Correspondence and reprint requests should be addressed to: Marie-Christine Rio, IGBMC, BP 10142, 67404 Illkirch CEDEX France. Phone: 333886534 24; fax: 333886532 01; e-mail: rio@igbmc.u-strasbg.fr. for TFF2, which is secreted by the mouse and pig pancreas but absent from human pancreas, their normal expression pattern is conserved across species (1-3). In addition to their distribution in the normal gut, TFFs can also be ectopically expressed in the damaged GI tract supporting the hypothesis that they mediate repair processes. It has been shown, in cell culture, that TFFs promote epithelial restitution after injury and protect the integrity of the epithelial barrier. Moreover, experiments using recombinant proteins in various animal models of gastric injury have demonstrated a role for TFF2 and TFF3 in processes of repair (5-8). Similarly, transgenic mice expressing TFF1 in their jejunum were more resistant to damage inflicted to this part of the GI tract (9). Using mice deficient for TFF3, Mashimo et al. (10) confirmed the protective role of TFF3, because these animals had an increased sensitivity to intestinal damage.

Disruption of TFF1 led to a drastic phenotype. All deficient mice developed antropyloric adenoma and occasionally multifocal intraepithelial or intramucosal carcinomas, suggesting a tumor suppressor function 
of TFF1 (11). TFF1 was originally identified as an estrogen-inducible gene in a breast cancer-derived cDNA library and aberrant expression of TFFl was detected in various human carcinomas. On the other hand, TFFl expression was found to be lost in $40-60 \%$ of gastric carcinomas while the normal adjacent tissue remained positive $(2,12)$. The three TFF genes are clustered within $40 \mathrm{~kb}$ of the mouse chromosome 17 (13) and within a region of $50 \mathrm{~kb}$ on human chromosome 21 q22.3 (14). Loss of heterozygosity studies showed that this region was commonly deleted in human gastric cancers $(15,16)$, suggesting that it bears one or several tumor suppressor genes. Recently, somatic mutations of the TFF1 gene have been reported in $16 \%$ of gastric cancers (17). Silencing of the TFFl gene expression via DNA hypermethylation of the TFFl promoter was also shown to occur in early stages of stomach carcinogenesis (18). In addition, treatments of GI cell lines with TFF1 have been shown to reduce cell proliferation $(19,20)$. Altogether, these results strongly suggest that TFF 1 is a gastric-specific tumor suppressor gene.

It has been shown for some time that TFFs and mucins are closely connected (21). In stomach, TFF1 is usually secreted in association with the MUC5AC mucin, and direct interaction has been demonstrated between the two proteins $(22,23)$. Therefore, it is likely that TFF1 participates in the correct organization of the mucus layer that protects the apical side of the gastric mucosa from deleterious luminal agents. Interestingly, TFFl null mice have been shown to have an altered mucus layer on their gastric mucosa indicating that alteration of the mucin expression and/or secretion might occur in these mice (11, and our unpublished results).

How TFFs mediate their function is not clear. Insights into the intracellular signaling events underlying the function of TFF3 have been reported. TFF3 treatment of human gastric or colon cell lines causes tyrosine phosphorylation of the epidermal growth factor receptor (EGF-R) and activation of mitogenactivated protein kinases (MAPK) $(24,25)$. In addition, TFF3 was found to prevent apoptosis in a PI3K-Akt dependent pathway $(25,26)$. Similar results were reported for TFF1 (27). Moreover, TFF3 has been shown to activate the NFkB pathway (28). The presence of specific trefoil receptors has not been completely proven yet, although recently, using a protein chemistry approach, a TFF2 binding protein has been isolated from porcine stomach mucosa. This protein was identified as the CRP-ductin gene product, a transmembrane protein expressed in intestinal crypt cells (29). CRP-ductin fulfills the criteria for being a TFF receptor, but it possesses no intrinsic enzymatic activity and, to date, no signaling pathway has been associated with this molecule.

In the present study, to further study the TFFl function, we investigated the molecular alterations occurring in response to TFF1 deficiency. Therefore, the molecular phenotype of the TFFl null antropyloric tumors was explored using the method of differential screening of a cDNA array. This technique provides a powerful approach to characterize at the molecular level cells or tissues because the expression of hundreds genes can be measured at a time (30). Based on the assumption that severe up-regulation of genes should occur subsequently to the lack of TFFl, we have differentially screened a collection of 588 well-characterized mouse cDNAs with either total or enriched probes prepared using the tumor tissue of TFF1 null mice and the corresponding normal tissue of wild-type mice.

\section{Materials and Methods \\ RNA Preparation and Analysis}

Pools of matching TFF1 null and wild-type mice were selected at 8 weeks and 1 year of age. The tumor region of TFF1 null mice and the normal corresponding antropyloric region of wild-type animals were dissected and immediately frozen in liquid nitrogen. Total RNA was extracted from the tissues using the $\mathrm{LiCl} /$ urea/acid phenol extraction method, followed by sodium acetate precipitation as described previously (4). Poly(A) ${ }^{+}$RNA was selected by two passages through an oligo(dT)-cellulose chromatography (31). Total RNAs were fractionated by electrophoresis on $1 \%$ agarose, $2.2 \mathrm{M}$ formaldehyde gels, transferred to nylon membrane (Hybond N, Amersham Corp., Arlington Heights, IL, USA), and immobilized by baking for $2 \mathrm{hr}$ at $80^{\circ} \mathrm{C}$.

\section{Screening Probe Preparation}

To obtain a tumor-specific total probe (total probe "plus"), $2 \mu \mathrm{g}$ of poly(A) ${ }^{+}$RNA was purified from the tumor region of five TFFl null mice at 8 weeks of age, subjected to first-strand cDNA synthesis using AMV reverse transcriptase (Roche Diagnostics GmbH, Mannheim, Germany) and oligo(dT) (Pharmacia Fine Chemicals, Piscataway, NJ, USA) as primer. An antropyloric control total probe (total probe "minus") was obtained similarly from $2 \mu \mathrm{g}$ of poly(A) ${ }^{+}$RNA extracted from five wild-type mice at 8 weeks of age. In both cases, after cDNA synthesis, RNA was removed by $\mathrm{NaOH}$ hydrolysis and cDNA was purified through Sephadex G50 chromatography (Pharmacia Biotech AB, Uppsala, Sweden) and concentrated by ethanol precipitation. For hybridization of cDNA array filters, $50 \mathrm{ng}$ of each single-strand cDNAs were ${ }^{32} \mathrm{P}$-labeled using random priming to $3 \times 10^{7} \mathrm{cpm}$ (total probe "plus") and to $2.7 \times 10^{7} \mathrm{cpm}$ (total probe "minus") using High prime DNA labeling system (Roche Diagnostics GmbH).

Enriched probes were obtained by subtractive hybridization as described previously (31). Briefly, $2 \mu \mathrm{g}$ of poly(A) ${ }^{+}$RNA extracted from tumor and normal antropyloric tissues were converted into 542 ng and 656 ng of single-strand cDNA, respectively. Complementary DNAs, "plus" and "minus" were 
hybridized for $24 \mathrm{hr}$ at $68^{\circ} \mathrm{C}$ to $7.5 \mu \mathrm{g}$ and $8.5 \mu \mathrm{g}$ of poly $(\mathrm{A})^{+}$RNA extracted from the normal antropyloric region of wild-type mice (15-fold excess), respectively. After hydroxylapatite chromatography, $15 \%$ and $20 \%$ of the cDNA remained single stranded, respectively. In both cases, single-stranded cDNAs were concentrated and washed with Tris $10 \mathrm{mM}$, EDTA $1 \mathrm{mM}$ buffer using Centricon 30 (Amicon, Beverly, MA, USA). ${ }^{32} \mathrm{P}$-random labeling of $10 \mathrm{ng}$ of single-stranded cDNAs gave $5 \times 10^{6} \mathrm{cpm}$ and $3.5 \times$ $10^{6} \mathrm{cpm}$ of enriched probe "plus" and enriched probe "minus," respectively.

\section{cDNA Array Hybridization and Analysis}

Atlas cDNA expression array from Clontech Laboratories (Palo Alto, CA, USA) was chosen for these studies. This array contains 588 independent immobilized mouse cDNAs that are spotted in duplicate on a nylon membrane and arranged in six panels noted from A to $\mathrm{F}$ by the manufacturer. Each panel contains 98 different characterized cDNAs. The cDNAs are spotted on the six panels according to their biological function (Table 1). A complete list of the genes included on the membranes is available (http://www.clontech.com). Hybridization and washing of the cDNA Array membranes were carried out according to the manufacturer instructions. Briefly, after labeling, probes were purified by G50 chromatography, and hybridization was carried out at $71^{\circ} \mathrm{C}$ in a rotation hybridization oven (model 45008, Bioblock Scientific, Illkirch, France) using $1 \times 10^{6} \mathrm{cpm} / \mathrm{ml}$ of radioactive probes for $12 \mathrm{hr}$. The membranes were then washed at $68^{\circ} \mathrm{C}$ four times with $2 \times$ standard sodium saline (SSC) and $1 \%$ sodium dodecyl sulfate (SDS), and then two times with $1 \times$ SSC, $0.1 \%$ SDS. Filters were exposed for 1-3 days and analyzed by phospho-imaging system. Stripping of the membranes was performed according to the manufacturer's instructions and filters were reused three times.

\section{Specific Probe Preparation}

Specific probes corresponding to the identified cDNAs were obtained by amplification by reverse transcription and polymerase chain reaction (RT-PCR), and subcloning into the pTadvantage vector (Clontech). The synthetic oligonucleotides used were as followed: for CHOP10 (Accession no. X67083), the AAS43 (GGAAGTGCAT CTTCATACAC CACC), and AAS44 (TGACTGGAAT CTGGAGAGCG AGGGC); for ERp72 (Accession no. J05186), the AAS51 (GAGAAGTTCC AGTCCAAGTA TGAGCC), and ABQ32 (CAAGATCCTG CCATTTCAC); for GRP78 (Accession no. D78645), the ABP107 (AGGATGCGGA CATTGAAGAC), and ABP108 (CAATTCCTCC TCTCCCTGAC); for 58-kDa inhibitor of RNAactivated protein kinase (p58IPK) (Accession no. U28423), the ABX54 (GTGCTCAAAT GTAACCCC) and ABX55 (GCTTTCTCTA AACCTTCCC); for Clusterin (Accession no. L08235), the AAN297 (TCTGGATGAA CGGCGACCGC ATCG) and AAN298
(CTGCGGACCA AGCGGGACTT GGG); for glyceraldehyde-3-phosphate dehydrogenase (GAPDH; Accession no. M32599), the PX24 (CCAGTATGAC TCCACTCACG GCAA) and PX25 (TATTCATTGT CATACCAGGA AATG). Probe specific for TFF1 corresponded to the complete cDNA (Accession no. NM009362).

\section{Electron Microscopy}

Four-month-old TFFl null and wild-type mice were anaesthetized and sacrificed by cardiac perfusion of a fixative containing $2.5 \%$ gluteraldehyde, $2 \%$ paraformaldehyde, and $0.2 \%$ tannic acid in $0.1 \mathrm{M}$ sodium cacodylate buffer, $\mathrm{pH}$ 7.4. The antral region of the stomach was diced into small pieces and immersed in the same fixative for about $3 \mathrm{hr}$ at $4^{\circ} \mathrm{C}$. After three washes in cacodylate buffer, the tissues were postfixed for $1 \mathrm{hr}$ in $1 \%$ osmium tetroxide partially reduced with potassium ferrocyanide. Tissue pieces were dehydrated in graded ethanol and embedded in Araldite. Tissue blocks were trimmed and semithin sections were stained with toluidine blue and examined under the light microscope for orientation and block trimming. Ultrathin sections were then cut, stained with uranyl-lead and finally examined under the Phillips electron microscope (C12-208 or CM10).

\section{Results}

Screening of a Mouse Atlas Array Detected Seven Up-regulated cDNAs in a TFF1 Null Antropyloric Tumor Total Probe

To identify genes involved in the development of the phenotype of the TFFl null mice, we performed a series of cDNA array hybridization experiments. In the first experiment, the mouse cDNA expression array was hybridized using total probes derived from the antropyloric tumor of 8-week-old TFF1 null mice (total probe "plus") and from the corresponding normal antropyloric region of matching wildtype mice (total probe "minus") (Fig. 1A). Only 10-20 cDNAs loaded on each panel (A-F) gave positive spots. The patterns of expression of these cDNAs were compared and seven genes belonging to four different panels (A, B, D, and E) were found to be up-regulated in the TFFl null antropyloric total probe "plus" (Fig. 2A).

Fifteen Additional Overexpressed cDNAs Were Detected

To improve the sensitivity of the detection, a second hybridization experiment was performed. Using subtractive hybridization (31), we synthesized enriched cDNA probes (32) (Fig. 1B). The enriched probe "plus," derived from the cDNAs of tumors of 8-week-old TFFl null mice, was deprived of sequences expressed in the normal corresponding tissue, namely the antropyloric region of 8-week-old wild-type mice. The enriched probe "minus" was 
Table 1. Characteristics of the 22 cDNAs found to be overexpressed in the antropyloric tumors of 8-week-old TFFl null mice

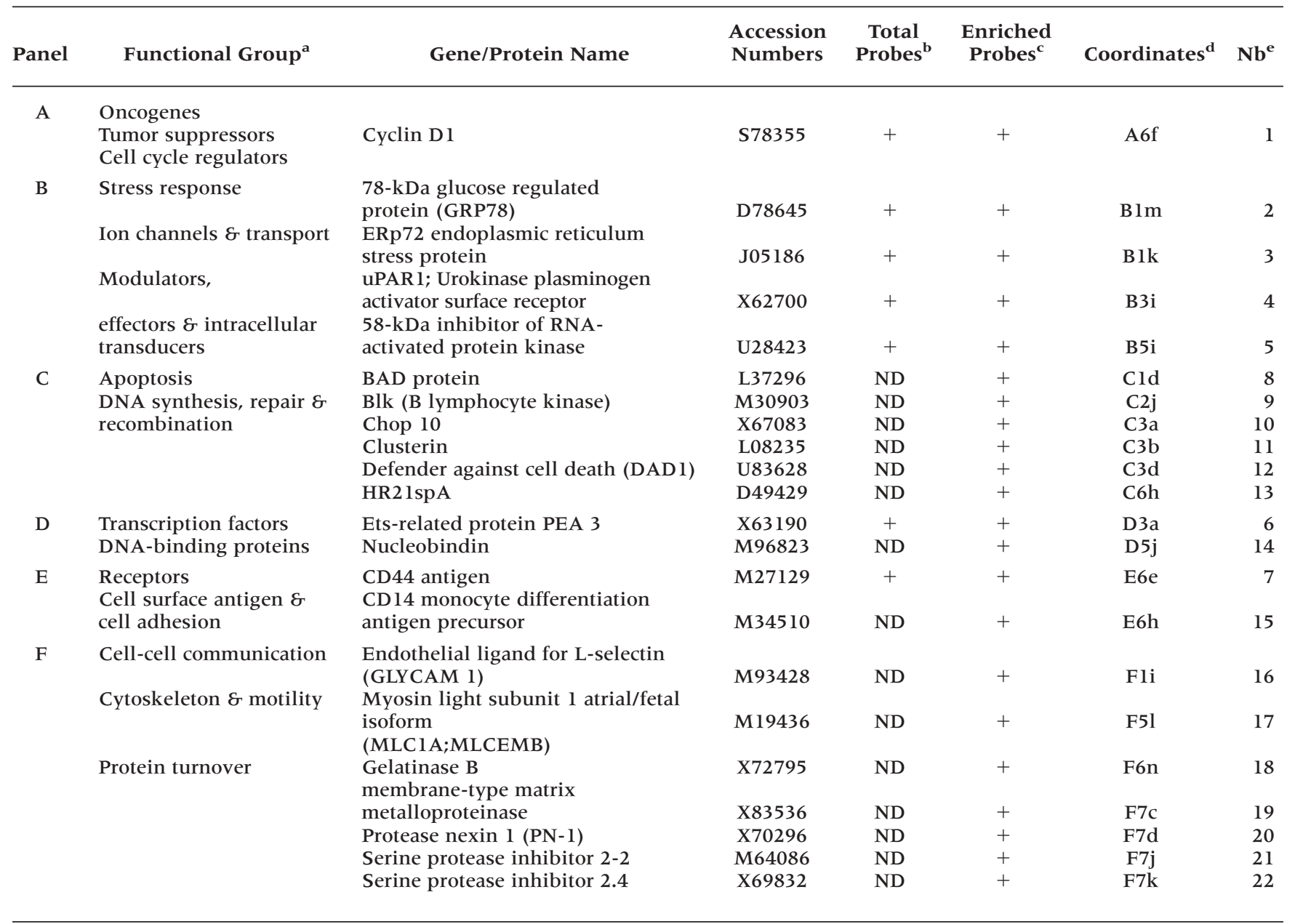

${ }^{a}$ The 588 mouse cDNAs immobilized in dots on nylon membrane are arranged by the manufacturer in 6 panels (A to F) in which several functional groups are represented

$\mathrm{b}+$, up-regulated genes; ND, no difference

c + , up regulated genes

${ }^{\mathrm{d}}$ Coordinates from the Atlas ${ }^{\mathrm{TM}}$ Mouse cDNA Expression Array (Clontech, Palo Alto, CA)

${ }^{\mathrm{e}}$ Identification numbers corresponding to Figure 2

similarly derived from normal antropyloric cDNAs (see Methods). Thus, the enriched probe "plus" contained genes expressed in the tumor and not in the normal antropyloric tissue and rare transcripts, while the enriched probe "minus" contained rare transcripts expressed in the normal antropyloric tissue. Hybridization of these two subtracted probes to the cDNA arrays gave 50-70 positive signals on each panel. Their comparison allowed the detection of 22 up-regulated genes in the enriched probe "plus," that included the seven genes found previously using total probes (Fig. 2B). Thus, 15 additional genes overexpressed in the TFF1 null tumors were detected by the method of screening using enriched probes. Together, our results indicated that 22 out of 588 (3.7\%) cDNAs loaded on the Atlas mouse cDNA expression array were overexpressed in the TFF1 null antropyloric tumor samples used to construct the probes.

Nature of the 22 Genes Up-regulated in the TFF1 Null Antropyloric Tumor Samples

The 22 genes overexpressed in the tumor samples of TFF 1 null mice were distributed in all array panels (Fig. 2A and 2B). Their identity was established according to their location on the array and the given manufacturer classification. As indicated in Table 1, they corresponded to: Cyclin D 1, a cell cycle regulator (panel A \#1); the 78-kDa glucose regulated protein (GRP78) and ERp72 endoplasmic reticulum (ER) stress protein, two proteins involved in stress response (panel B \#2 and 3); the urokinase plasminogen activator surface receptor 


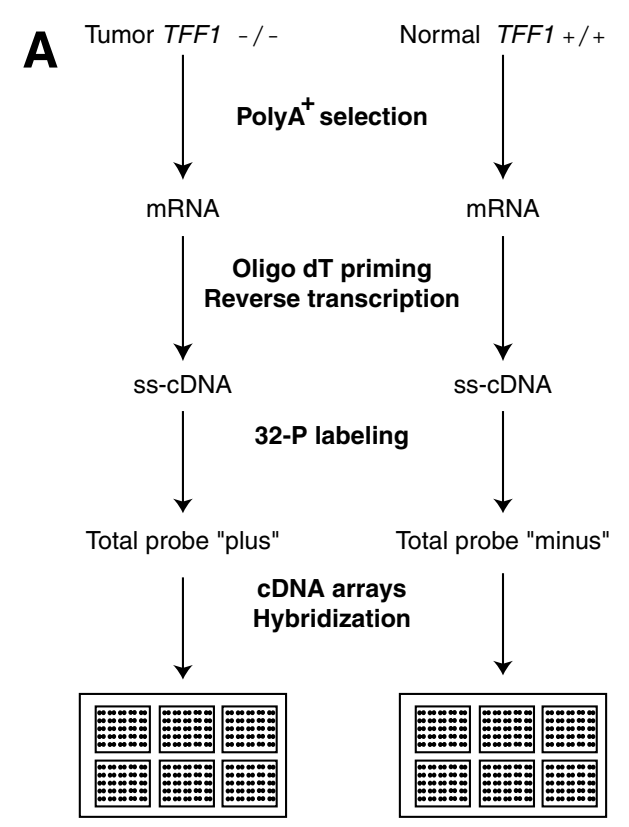

Signal comparison

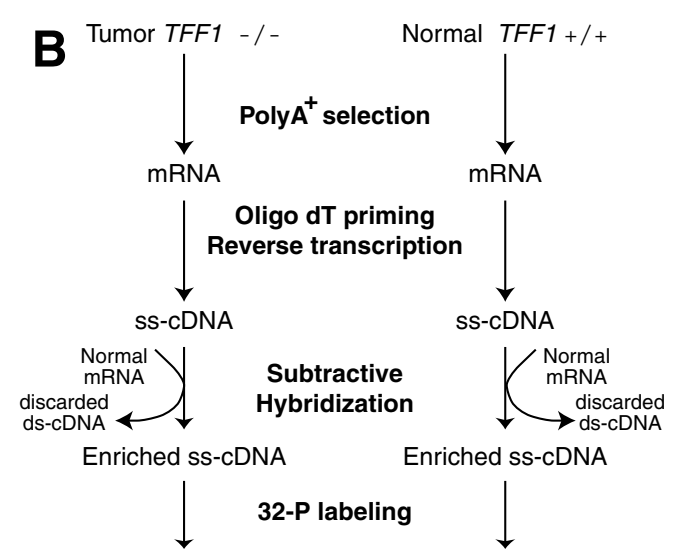

Enriched probe "plus" $\quad$ Enriched probe "minus"

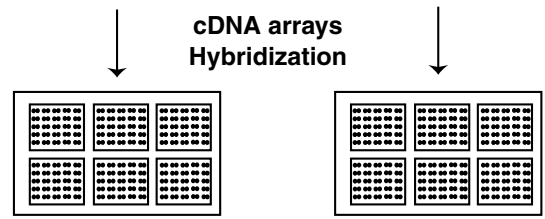

Signal comparison

Fig. 1. Scheme of the strategy used for the screening of the Atlas mouse cDNA expression array. Total (A) and enriched (B) probes were obtained using normal $(\mathrm{TFF} 1+/+)$ and tumor $(\mathrm{TFF} 1-/-)$ antropyloric tissues from 8 -week-old mice.

(UPARl) and 58-kDa inhibitor of RNA-activated protein kinase (p58IPK), two genes classified as modulators, effectors, and intracellular transducers (panel B \# 4 and 5); BAD, B lymphocyte kinase
(Blk), CHOP 10, Clusterin and Defender against cell death (DAD1), five genes linked to apoptosis (panel C \#8, 9, 10, 11, and 12); HR21spA, a gene involved in DNA repair (panel C \#13); PEA3, an
A Total probe "plus"

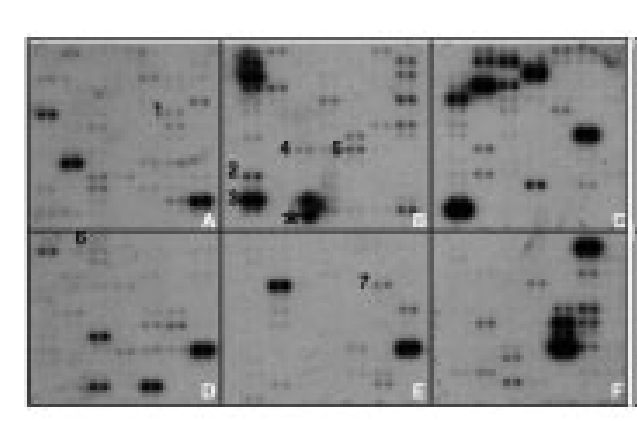

Total probe "minus"

Normal antro-pyloric TFF1 +/+

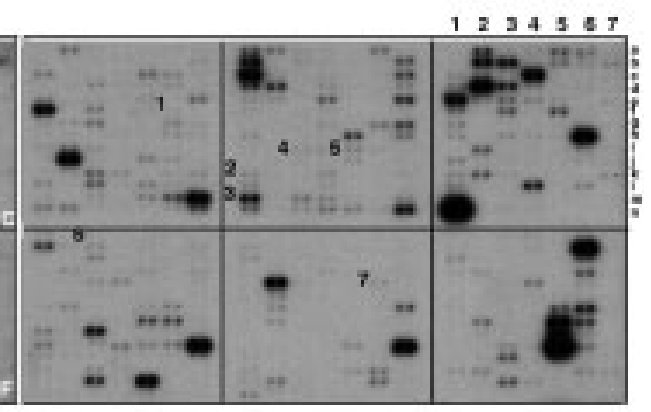

B Enriched probe "plus" Tumor antro-pyloric TFF1 - /-

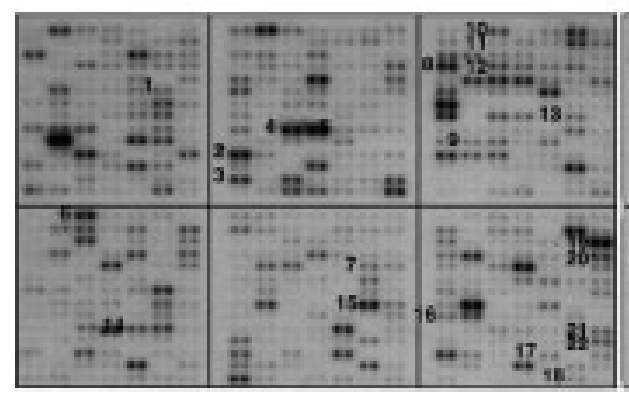

Enriched probe "minus"

Normal antro-pyloric TFF1 + /+

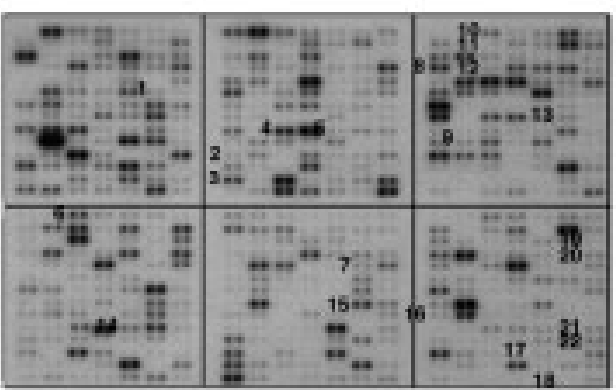

Fig. 2. Representative mouse Atlas cDNA expression array analysis. The cDNA hybridization patterns using the "plus" (TFF1-/-) and "minus" (TFFI $+/+$ ) total probes (A), or enriched probes (B) are shown. The numbers (1-22) located on the left of dots identify the position of the cDNAs up-regulated in the TFF1 null antropyloric tumors. *Background spot. Panels (white letters) and dot coordinates (numbers and small letters) are indicated. 
Ets-related protein and Nucleobindin, two DNA binding proteins (panel D \#6 and 14); CD44 and CD 14, two cell surface antigens (panel E \#7 and 15); the endothelial ligand for L-selectin (GLYCAM 1) (panel F \#16); Myosin light subunit atrial/fetal isoform (MLC1A), a cytoskeleton protein (panel F \# 17); gelatinase B (Gel B), membrane-type matrix metalloproteinase (MTl-MMP), Protease nexin 1 $(\mathrm{PN}-1)$, serine protease inhibitor 2-2 and serine protease inhibitor 2.4, five genes involved in cell-cell communication, cytoskeleton, and motility (panel F \# 18, 19, 20, 21 and 22). Several of these genes have already been shown to be involved in tumor processes in human and mouse (33-37). However, we observed overexpression of GRP78 and ERp72, an established "readout" of the unfolded protein response (UPR) pathway (38-41). Moreover, bibliographic searches showed that, among the other identified genes, p58IPK (42), CHOP10 (43), and Clusterin (44) were also molecules involved in this ER stress-mediated process. These results suggested a possible link between TFF 1 deficiency and UPR. Therefore, we decided to confirm the expression of these five genes in TFF1 null antropyloric tumors.

\section{UPR Activation Is a Common and Permanent Feature of TFF1 Null Antropyloric Tumors}

To investigate if GRP78, ERp72, p58IPK, CHOP 10 and Clusterin overexpression observed by array screening was shared by all the antropyloric tumors developed in TFF1 null mice, their expression was further studied using Northern blot experiments. Total RNAs prepared from the tumor region of 3 TFFl null mice and from the corresponding normal antropyloric region from three wild-type mice were analyzed using specific probes obtained by RT-PCR amplification (see Materials). TFFl and GAPDH probes were used as controls. We first studied tumors developed in 8-weekold TFFl-deficient mice. As expected, the five genes were overexpressed in all tumors studied, but not in the corresponding normal tissues nor in wild-type and TFF1 null intestine, a tissue that normally does not express TFFl (Fig. 3, lanes 1 and 2, and data not shown). Thus, activated UPR characterizes antropyloric tumors developed in 8-week-old TFF1 null mice.

The expression of GRP78, ERp72, p58IPK, CHOP10, and Clusterin was then studied in tumors from 1-year-old mice. All genes remained overexpressed at this advanced age. The differential expression ratio between normal and tumor tissues was similar to or higher than that observed at 8 week of age (Fig. 3, lanes 3 and 4), indicating that these genes are not specific of one particular step of tumorigenesis. As previously observed in 8-week-old mice, TFF1 null intestine did not show any differential expression (data not shown). Finally, in 1-year-old tumors, Clusterin showed a dramatic up-regulation of its transcription, leading to about 100 -fold increased mRNA level (Fig. 3, lane 4). This suggests a

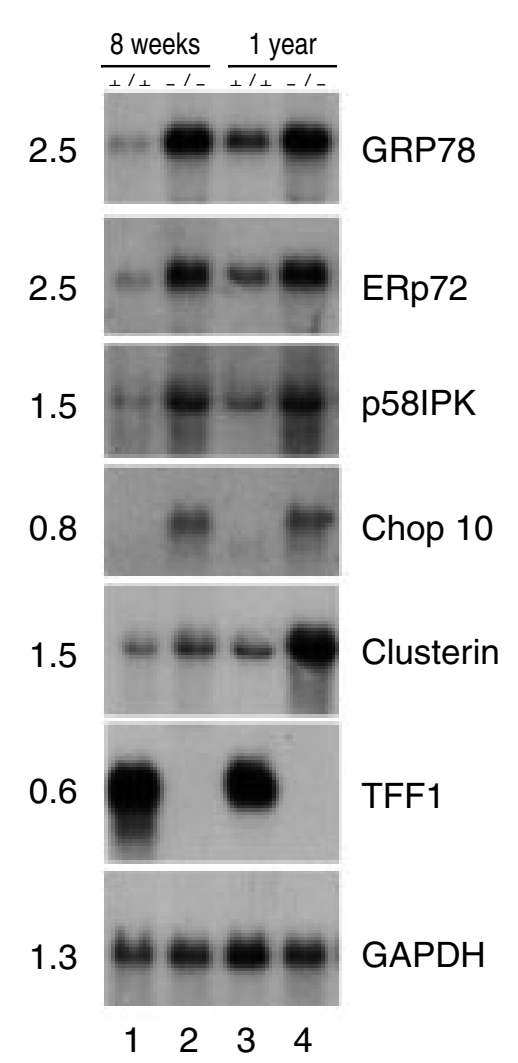

Fig. 3. Northern blot analysis of GRP78, ERp72, p58IPK, CHOP10, and Clusterin mRNAs in antropyloric mouse tissues. Each lane contained $10 \mu \mathrm{g}$ of total RNA. Lanes 1 and 3, RNA samples from normal tissues from 8-week-old and 1-yearold wild-type $(\mathrm{TFF} 1+/+$ ) mice, respectively. Lanes 2 and 4 , RNA samples from antropyloric tumors from 8-week-old and 1-year-old null (TFF1-/-) mice, respectively. Hybridization was carried out using ${ }^{32} \mathrm{P}$ cDNA probes. The GAPDH probe was used as a positive internal loading control. The TFF1 probe was used as control of TFF1 null mutation.

preponderant function for Clusterin in advanced tumors. Together, these results showed that UPR is not a transiently activated process during tumorigenesis, but represents a constant and permanent feature of TFF1 null antropyloric tumors.

\section{Epithelial Cells of TFF1 Null Antropyloric Tumors Showed Enlarged and Overloaded Rough ER}

Because UPR is induced by ER stress, we looked for abnormalities of the rough ER of TFFl null antropyloric epithelial cells. Electron microscopic examination of the glandular epithelial cells of the pyloric antrum of normal mice showed the two normal mucous cell lineages and their early progenitors: pre-pit and pregland cells (45) (data not shown). Examination of the antrum in TFFl null mice revealed an increase in the thickness of the mucosa due to epithelial hyperplasia and in some places there was an additional loss of the tubular glandular pattern (adenoma) confirming our previous light microscopic observations (11). Detailed examination of the hyperplastic cells revealed an 

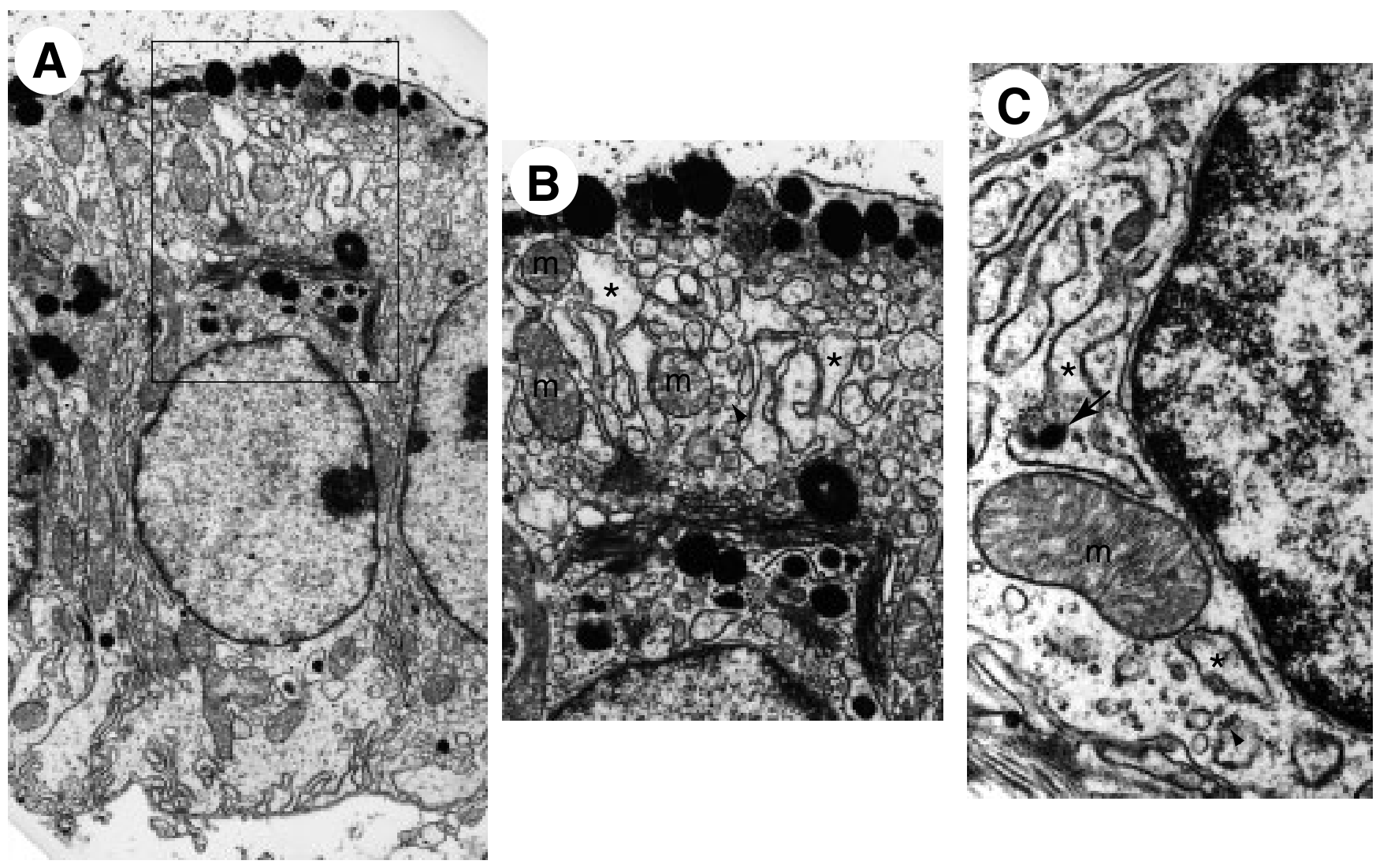

Fig. 4. Ultrastructural analysis of antropyloric tumor cells of TFF1 null mice. Electron micrographs of (A) pre-pit cell cut through apex and base (respectively seen at the top and bottom) and attached to two neighbor cells partially seen on the sides. The cell is characterized by an euchromatic central nucleus and a prominent supranuclear Golgi apparatus producing homogeneously dense mucous granules. In addition, some secretory granules are also visible at the apical surface (original magnification $\times 4000$ ). (B) High magnification of the area within the rectangle seen in (A) showing details of apical cytoplasm. Note that in between the Golgi region and the apical secretory granules, the cytoplasm is full of dilated cisternae of rough ER (asterisks). Few mitochondria $(\mathrm{m})$ are scattered (original magnification $\times 6300$ ). (C) A close up of another gastric epithelial cell showing part of the nucleus seen at the right and dilated cisternae of rough ER. Arrowhead, example of ribosomes of the rough ER. Note the accumulation of dense secretory material (arrow) within the lumen of ER (original magnification $\times 16,000$ ).

apparent alteration in the machinery of protein synthesis. Dilatation of the cisternae of rough ER (Fig. 4A and $4 \mathrm{~B}$ ) and accumulation of dense secretory material in their lumen (Fig. 4C) were observed in the hyperplastic epithelial glandular cells of the pyloric antrum. Thus, the ultrastructural structure of the rough ER, as typically observed in UPR, is altered in response to TFFI deficiency.

\section{Discussion}

Loss or alteration of TFFl gene expression occurs in human gastric cancers suggesting that TFFl is a tumor suppressor gene (reviewed in 13). Accordingly TFF1 null mice develop antropyloric tumors (11). The present study was undertaken to investigate in the mouse tumors the molecular mechanism altered in response to TFFI deficiency.

Reassociation profiles have shown that a given cell or tissue expresses about 15,000 distinct genes distributed in three classes: abundant, intermediate, and rare. Each account for approximately $22 \%, 41 \%$, and $37 \%$ of total transcripts and represent approximately 10, 840, and 14200 distinct genes, respectively (46). Using total probes established using normal or tumor antropyloric tissues, most of the cDNAs present on the array did not show any signal, indicating that these probes only permitted the detection of the abundant transcripts. Therefore we decided to repeat the same experiment using enriched probes (32), which are representative of differentially expressed genes. Because we were mainly interested in the genes up-regulated in the tumors, we enriched the cDNA probe "plus" in genes expressed in the TFFl null tumors by subtractive hybridization with normal antropyloric mRNA. The hybridization profile of this enriched probe "plus" was compared to a control probe that was referred as enriched probe "minus." Using these two probes, most of the genes present in the array were visualized. Together, these results demonstrated that screening a cDNA expression array with enriched probes allows the 
study of transcripts of the intermediate and rare abundance classes, and therefore gives a better definition of the gene expression profile of a given set of tissues.

Array screenings led to the identification of 22 genes overexpressed in the tumors of TFF1 null mice, 15 of them being only detected using the enriched probes. As expected for a tumor tissue, a subset of identified genes encode proteins involved in regulating the balance between cell proliferation and cell death such as Cyclin D1, Bad, and PEA3 $(36,37)$. Several others corresponded to genes related to cell-cell and/or cell-matrix relationship. Thus, during tumorigenesis, MMPs, serine proteinases, and their inhibitors are transiently expressed proteins involved in a proteolytic cascade leading to matrix remodeling (33-35). Consistently, the TFF1 null antropyloric tumors overexpressed two MMPs, the Gel B and MTl-MMP, the serine protease receptor UPAR, and three serine protease inhibitors (serpins). The identification of these genes already known to participate in tumor processes validated our approach taken to identify genes that might be specifically deregulated in antropyloric tumors in response to TFFl deficiency.

Alteration of another set of genes encoding for proteins related to the ER machinery was more intriguing. One of the functions of the ER is to control the processing of secretory (membrane and secreted) proteins. These proteins are translocated into the ER lumen, which serves as a site of protein folding, assembly, and degradation (47). Folding reactions are mediated by a set of specific resident ER luminal proteins (38-41). TFF1 deficiency causes an impressive transcriptional induction of two such proteins, GRP78 and ERp72. The GRP78 (also named BiP) is a member of the family of heat shock protein 70 (HSP70) molecular chaperones. It binds permanently to misfolded, underglycosylated, or unassembled proteins whose transport from the ER is blocked. It is also essential for the transport back across the membrane of aberrant polypeptides destined for degradation by the proteasome (48). In addition, it exhibits an anti-apoptotic function in stress conditions, such as tumor growth (49). ERp72 belongs to the protein disulphide isomerase family and promotes correct protein folding in a sequential or simultaneous manner with GRP78. Overexpression of GRP78 and ERp72 is synonymous with the adaptive cellular response called UPR (38-40). In addition, we observed an up-regulation of p58IPK, a tetratricopeptide repeat-containing co-chaperone protein known to be involved in stress-activated cellular pathways. p58IPK also has anti-apoptotic and oncogenic properties (42). Finally, GRP78, ERp72, and p58IPK overexpression was observed in antropyloric tumors whatever the time of observation (8 weeks or 1 year), indicating that their transcriptional up-regulation and therefore the UPR activation was not related to a specific stage of tumor development but to the lack of TFF1 expression. Together, these data clearly indicate that TFFl deficiency permanently activates UPR cellular process in TFF1 null antropyloric tumors.

In this context, two non-ER resident proteins, CHOP10 (also named GADD153) and Clusterin, known to play a role in UPR, were also up-regulated in TFF1 null antropyloric tumors. Thus, it has been reported that defective function of the ER triggers the induction of CHOP10. CHOP10 is a C/EBP homologous transcription factor whose expression occurs primarily in response to the presence of unfolded proteins in the ER (43). It is an effector of the response of cells to ER overload. CHOP10 also has a pro-apoptotic function that plays a crucial role in the induction of cell death under conditions associated with malfunctions of the ER. Finally, we identified Clusterin that is a secreted protein expressed by epithelial cells of many organs. Although extensively studied, Clusterin, also named testosterone, repressed prostate messenger 2 (TRPM2), dimeric acidic glycoprotein (DAG), apolipoprotein J (Apo J), or sulfated glycoprotein 2, has no clear established biological function. It has been implicated in lipid transport, reproduction, complement regulation, tissue remodeling, apoptosis, and cell survival. More recently, it has been shown that Clusterin is a stress-induced heat shock protein with cytoprotective abilities. Clusterin can stabilize stressed proteins in a refolding competent state (44). As for GRP78, ERp72, and p58IPK, CHOP10 and Clusterin overexpression was permanent in TFFl null tumors.

Together, our data indicate that TFF1 deficiency leads to UPR activation. UPR arises in several noxious circumstances including calcium depletion, viral infection, and ischemia (38-41), but none of these occur in TFF1 null tumors. It has been reported that, whatever the stimulus, the common feature of UPR activation is the accumulation of misfolded proteins in the ER (38-41). Consistently, ultrastructural analysis showed that the lumen of the ER of the epithelial antropyloric cells of the TFF1 null mice exhibited an enlarged structure characteristic of ER protein accumulation. Thus, TFFl deficiency leads to ER accumulation of proteins, and it can be hypothesized that TFFl plays a role in the ER machinery. This calls into question the possible function of TFFl in the folding and/or secretory processes themselves. In this context, TFF1 null mice exhibit altered mucus layer along their gastric mucosa (11). Moreover, human gastric carcinomas express aberrant mucins (50). In vitro, TFFl has been shown to directly bind to mucins $(22,23)$. As secreted molecules, both TFF1 and mucins transit through the ER. Thus, in vivo TFF1/mucin binding could occur inside the ER favoring correct mucin folding and/or secretion. Such a function in protein maturation has already been proposed for TFFl because TFFl is not only copackaged and cosecreted in mucous granules, but also in neuroendocrine granules, which have different functions (51). 
In conclusion, our findings link for the first time a member of the TFF family to the ER signaling pathway. In this context, it is interesting to note that the mice knocked out for the IREl $\beta$ (inositol requiring) gene, the gut-specific sensor of ER stress (38-41), gives a phenotype similar to that observed in mice deficient for TFF3 (10), another member of the TFF family. Thus, mice lacking IRE $1 \beta$ develop colitis of increased severity, suggesting a link between the handling of the ER stress and susceptibility to environmental agents that promote colitis (52). To date TFFl is viewed as an extracellular factor thought to act via an as yet unidentified specific membrane receptor. Accordingly, addition of recombinant TFF1 to the culture medium of GI cells provides protection from induced apoptosis $(19,20)$. It is therefore tempting to speculate that TFFl acts both intracellularly and extracellularly to protect gastric cells from stress.

\section{Acknowledgments}

We thank F. Ruffenach, I. Colas, and E. Troesch for their technical assistance, and C. Nourse for critical reading of the manuscript. L.F.T. was a recipient of a fellowship from the Instituto Nacional de Ciencias Médicas y de la Nutricion "Salvador Zubiran" (INCMNSZ), the Instituto de Investigaciones Biomédicas, UNAM, and the Consejo Nacional de Ciencia y Tecnologia (CONACYT). This work was supported by funds from the Institut National de la Santé et de la Recherche Médicale, the Centre National de la Recherche Scientifique, the Hôpital Universitaire de Strasbourg, the Association pour la Recherche sur le Cancer, the Ligue Nationale Française contre le Cancer and the Comités du HautRhin et du Bas-Rhin and the Fondation de France.

\section{References}

1. Thim L. (1997) Trefoil peptides: from structure to function. Cell Mol. Life Sci. 53: 888-903.

2. Ribieras S, Tomasetto C, Rio MC. (1998) The pS2/TFF1 trefoil factor, from basic research to clinical applications. Biochim. Biophys. Acta 1378: F61-F77.

3. Hoffmann W, Jagla W, Wiede A. (2001) Molecular medicine of TFF-peptides: from gut to brain. Histol. Histopathol. 16: 319-334.

4. Rio MC, Bellocq JP, Daniel JY, et al. (1988) Breast cancerassociated pS2 protein: synthesis and secretion by normal stomach mucosa. Science 241: 705-708.

5. Poulsen SS, Thulesen J, Nexo E, Thim L. (1998) Distribution and metabolism of intravenously administered trefoil factor 2/porcine spasmolytic polypeptide in the rat. Gut 43: 240-247.

6. Chinery R, Playford RJ. (1995) Combined intestinal trefoil factor and epidermal growth factor is prophylactic against indomethacin-induced gastric damage in the rat. Clin. Sci. (Colch.) 88: 401-403.

7. Babyatsky MW, de Beaumont M, Thim L, Podolsky DK. (1996) Oral trefoil peptides protect against ethanol- and indomethacin-induced gastric injury in rats [see comments]. Gastroenterology 110: 489-497.

8. Tran CP, Cook GA, Yeomans ND, Thim L, Giraud AS. (1999) Trefoil peptide TFF2 (spasmolytic polypeptide) potently accelerates healing and reduces inflammation in a rat model of colitis. Gut 44: 636-642.
9. Playford RJ, Marchbank T, Goodlad RA, Chinery RA, Poulsom R, Hanby AM. (1996) Transgenic mice that overexpress the human trefoil peptide pS2 have an increased resistance to intestinal damage. Proc. Natl. Acad. Sci. U.S.A. 93: 2137-2142.

10. Mashimo H, Wu DC, Podolsky DK, Fishman MC. (1996) Impaired defense of intestinal mucosa in mice lacking intestinal trefoil factor [see comments]. Science 274: 262-265.

11. Lefebvre O, Chenard MP, Masson R, et al. (1996) Gastric mucosa abnormalities and tumorigenesis in mice lacking the pS2 trefoil protein [see comments]. Science 274: 259-262.

12. Machado JC, Nogueira AM, Carneiro F, Reis CA, SobrinhoSimoes M. (2000) Gastric carcinoma exhibits distinct types of cell differentiation: an immunohistochemical study of trefoil peptides (TFF1 and TFF2) and mucins (MUC1, MUC2, MUC5AC, and MUC6). J. Pathol. 190: 437-443.

13. Ribieras S, Lefebvre O, Tomasetto C, Rio MC. (2001) Mouse Trefoil factor genes: genomic organization, sequences and methylation analyses. Gene 266: 67-75.

14. Seib T, Blin N, Hilgert K, et al. (1997) The three human trefoil genes TFF1, TFF2, and TFF3 are located within a region of $55 \mathrm{~kb}$ on chromosome 21q22.3. Genomics 40: 200-202.

15. Sakata K, Tamura G, Nishizuka S, et al. (1997) Commonly deleted regions on the long arm of chromosome 21 in differentiated adenocarcinoma of the stomach. Genes Chromosomes Cancer 18: 318-321.

16. Nishizuka S, Tamura G, Terashima M, Satodate R. (1998) Loss of heterozygosity during the development and progression of differentiated adenocarcinoma of the stomach. J. Pathol. 185: 38-43.

17. Park WS, Oh RR, Park JY, et al. (2000) Somatic mutations of the trefoil factor family 1 gene in gastric cancer. Gastroenterology 119: 691-698.

18. Fujimoto J, Yasui W, Tahara H, Tahara E, Kudo Y, Yokozaki H. (2000) DNA hypermethylation at the pS2 promoter region is associated with early stage of stomach carcinogenesis. Cancer Lett. 149: 125-134.

19. Calnan DP, Westley BR, May FE, Floyd DN, Marchbank T, Playford RJ. (1999) The trefoil peptide TFFl inhibits the growth of the human gastric adenocarcinoma cell line AGS. J. Pathol. 188: 312-317.

20. Bossenmeyer-Pourié C, Kannan R, Ribieras S, et al. (2002) The Trefoil factor 1 participates in gastrointestinal cell differentiation by delaying Gl-S phase transition and reducing apoptosis. J. Cell Biol. 157: 761-770.

21. Longman RJ, Douthwaite J, Sylvester PA, et al. (2000) Coordinated localisation of mucins and trefoil peptides in the ulcer associated cell lineage and the gastrointestinal mucosa. Gut 47: 792-800.

22. Tomasetto C, Masson R, Linares JL, et al. (2000) pS2/TFF1 interacts directly with the VWFC cysteine-rich domains of mucins. Gastroenterology 118: 70-80.

23. Newton JL, Allen A, Westley BR, May FE. (2000) The human trefoil peptide, TFF 1 , is present in different molecular forms that are intimately associated with mucus in normal stomach. Gut 46: 312-320.

24. Taupin D, Podolsky DK. (1999) Mitogen-activated protein kinase activation regulates intestinal epithelial differentiation. Gastroenterology 116: 1072-1080.

25. Kinoshita K, Taupin DR, Itoh H, Podolsky DK. (2000) Distinct pathways of cell migration and antiapoptotic response to epithelial injury: structure-function analysis of human intestinal trefoil factor. Mol. Cell. Biol. 20: 4680-4690.

26. Taupin DR, Kinoshita K, Podolsky DK. (2000) Intestinal trefoil factor confers colonic epithelial resistance to apoptosis. Proc. Natl. Acad. Sci. U.S.A. 97: 799-804.

27. Emami S, Le Floch N, Bruyneel E, et al. (2001) Induction of scattering and cellular invasion by trefoil peptides in src- and RhoA-transformed kidney and colonic epithelial cells. FASEB J. 15: 351-361.

28. Chen YH, Lu Y, De Plaen IG, Wang LY, Tan XD. (2000) Transcription factor NF-kappaB signals antianoikic function of trefoil factor 3 on intestinal epithelial cells. Biochem. Biophys. Res. Commun. 274: 576-582. 
29. Thim L, Mortz E. (2000) Isolation and characterization of putative trefoil peptide receptors. Regul. Pept. 90: 61-68.

30. Ferea TL, Brown PO. (1999) Observing the living genome. Curr. Opin. Genet. Dev. 9: 715-722.

31. Tomasetto C, Regnier C, Moog-Lutz C, et al. (1995) Identification of four novel human genes amplified and overexpressed in breast carcinoma and localized to the q11-q21.3 region of chromosome 17. Genomics 28: 367-376.

32. Sagerstrom CG, Sun BI, Sive HL. (1997) Subtractive cloning: past, present, and future. Annu. Rev. Biochem. 66: 751-783.

33. McCawley LJ, Matrisian LM. (2001) Matrix metalloproteinases: they're not just for matrix anymore! Curr. Opin. Cell. Biol. 13: 534-540.

34. Coussens LM, Werb Z. (1996) Matrix metalloproteinases and the development of cancer. Chem. Biol. 3: 895-904.

35. Conese M, Blasi F. (1995) Urokinase/urokinase receptor system: internalization/degradation of urokinase-serpin complexes: mechanism and regulation. Biol. Chem. Hoppe Seyler 376: 143-155.

36. Malumbres M, Barbacid M. (2001) To cycle or not to cycle: a critical decision in cancer. Nature Rev. Cancer 1: 223-231.

37. Fisher DE. (1994) Apoptosis in cancer therapy: crossing the threshold. Cell 78: 539-542.

38. Patil C, Walter P. (2001) Intracellular signaling from the endoplasmic reticulum to the nucleus: the unfolded protein response in yeast and mammals. Curr. Opin. Cell. Biol. 13: 349355.

39. Kaufman RJ. (1999) Stress signaling from the lumen of the endoplasmic reticulum: coordination of gene transcriptional and translational controls. Genes Dev. 13: 1211-1233.

40. Ng DT, Spear ED, Walter P. (2000) The unfolded protein response regulates multiple aspects of secretory and membrane protein biogenesis and endoplasmic reticulum quality control. J. Cell. Biol. 150: 77-88.

41. Ma Y, Hendershot LM. (2001) The unfolding tale of the unfolded protein response. Cell 107: 827-830.
42. Tang NM, Korth MJ, Gale M Jr, et al. (1999) Inhibition of double-stranded RNA- and tumor necrosis factor alphamediated apoptosis by tetratricopeptide repeat protein and cochaperone P58(IPK). Mol. Cell. Biol. 19: 4757-4765.

43. Wang XZ, Lawson B, Brewer JW, et al. (1996) Signals from the stressed endoplasmic reticulum induce C/EBP-homologous protein (CHOP/GADD 153). Mol. Cell. Biol. 16: 4273- 4280.

44. Wilson MR, Easterbrook-Smith SB. (2000) Clusterin is a secreted mammalian chaperone. Trends Biochem. Sci. 25: 95-98.

45. Karam SM. (1999) Lineage commitment and maturation of epithelial cells in the gut. Front. Biosci. 4: D286-D298.

46. Hastie ND, Bishop JO. (1976) The expression of three abundance classes of messenger RNA in mouse tissues. Cell 9: 761-774.

47. Kuznetsov G, Bush KT, Zhang PL, Nigam SK. (1996) Perturbations in maturation of secretory proteins and their association with endoplasmic reticulum chaperones in a cell culture model for epithelial ischemia. Proc. Natl. Acad. Sci. U.S.A. 93: 8584-8589.

48. Gething MJ, Sambrook J. (1992) Protein folding in the cell. Nature 355: 33-45.

49. Jamora C, Dennert G, Lee AS. (1996) Inhibition of tumor progression by suppression of stress protein GRP78/BiP induction in fibrosarcoma B/C10ME. Proc. Natl. Acad. Sci. U.S.A. 93: 7690-7694.

50. Perrais M, Pigny P, Buisine MP, Porchet N, Aubert JP, Van Seuningen-Lempire I. (2001) Aberrant expression of human mucin gene MUC5B in gastric carcinoma and cancer cells. Identification and regulation of a distal promoter. J. Biol. Chem. 276: 15386-15396.

51. Wright NA, Poulsom R, Stamp G, et al. (1993) Trefoil peptide gene expression in gastrointestinal epithelial cells in inflammatory bowel disease. Gastroenterology 104: 12-20.

52. Bertolotti A, Wang X, Novoa I, et al. (2001) Increased sensitivity to dextran sodium sulfate colitis in IRElbeta-deficient mice. J. Clin. Invest. 107: 585-593. 